\title{
Spectrally Constrained NIR tomography for Breast Imaging: Simulations and Clinical Results
}

\author{
Subhadra Srinivasan*, Brian W. Pogue, Shudong Jiang, \\ Hamid Dehghani, Keith D. Paulsen \\ Thayer School of Engineering, Dartmouth College, Hanover NH 03755
}

\begin{abstract}
A multi-spectral direct chromophore and scattering reconstruction for frequency domain NIR tomography has been implemented using constraints of the known molar spectra of the chromophores and a Mie theory approximation for scattering. This was tested in a tumor-simulating phantom containing an inclusion with higher hemoglobin, lower oxygenation and contrast in scatter. The recovered images were quantitatively accurate and showed substantial improvement over existing methods; and in addition, showed robust results tested for up to 5\% noise in amplitude and phase measurements. When applied to a clinical subject with fibrocystic disease, the tumor was visible in hemoglobin and water, but no decrease in oxygenation was observed, making oxygen saturation, a potential diagnostic indicator.
\end{abstract}

\section{INTRODUCTION}

Near Infra-red (NIR) tomography is a growing area of research in medical imaging, principally due to the functional nature of the information this modality provides. Light is absorbed by the hemoglobin and water in tissue, and in the NIR wavelength region (600-1000nm) this absorption is low enough to allow penetration through a few centimeters of tissue $^{1}$. Though scattering dominates, making it difficult to separate the two, measurement techniques and model-based reconstruction schemes have allowed the field to overcome this limitation and obtain concentrations of tissue chromophores and scatter-based parameters.

In breast imaging, this is of potential diagnostic value since tumors, show increased absorption ${ }^{2}$, due to the localized increase in total hemoglobin. Additional factors such as dense fibrosis and cell proliferation show contrast in scatter; and water may give a measure of the extravascular space ${ }^{4}$. Oxygen saturation obtained using concentrations of de-oxy and oxyhemoglobin, relate to the $\mathrm{pO}_{2}$ of oxygen in tissue, and this helps assess the hypoxia in tumors, critical in separating malignancies from benign tumors ${ }^{5,6}$. The current clinical modality of choice is mammography and suffers from high-false positive rates and the cumulative risk of a false positive result is $49.1 \%$ after 10 mammograms ${ }^{7}$. Overall, NIR has a niche in the evaluation of cancers, due to its ability to complement information from other modalities such as mammography and MRI, which are structure-based.

NIR tomography has emerged possible due to two principal areas of technological development, including NIR instrumentation and capabilities for diffuse image reconstruction. Advances in instrumentation have enabled multiwavelength measurements from time-domain based ${ }^{8}$ or frequency-domain based ${ }^{9}$ systems to be obtained with percent noise established around $1 \%$. In addition continuous-wave measurements ${ }^{10}$ exist, which allow broadband interrogation with intensity measurements, but no phase information, making the separation of absorption and scatter difficult. The current system built at Dartmouth and documented in McBride et $\mathrm{al}^{9}$ is a multi-wavelength frequency domain instrument allowing amplitude and phase measurement along the periphery of the breast. Having obtained this data, the second area of focus, which is pursued in this paper, is image reconstruction. This takes several forms ${ }^{11}$ such as analytical solutions for homogeneous geometries, statistical methods such as Monte Carlo and numerical approaches such as finite element and finite difference models. Initially images of absorption and scatter at single wavelengths were used to establish contrasts ${ }^{12}$, using some of these methods, and this was followed by multi-wavelength fitting at sufficient wavelengths to yield images of total hemoglobin, oxygen saturation, water ${ }^{13}$, and scatter-based parameters ${ }^{14}$.

*E-mail: Subha@dartmouth.edu; Tel: (603) 646-2859; Fax (603) 646-3856 
Next were quantification improvement schemes using techniques relating to regularization ${ }^{15}$, contrast-to-noise measures ${ }^{16}$ and second derivative ${ }^{17}$ to establish accuracy. The most recent technique ${ }^{18-20}$ which has been a breakthrough in terms of quantitative accuracy is the reconstruction for direct images of chromophore concentrations and scatter without intermediate recovery of optical properties, by usage of all available wavelengths of data simultaneously. This is achieved by incorporating the spectral behavior of these chromophores and scattering into the image-formation process as apriori information. Applied to continuous-wave data ${ }^{18}, 19$, with suitable assumptions on scatter, results have shown optimized images with lower noise and reduced cross-talk between the parameters.

We have implemented this type of spectrally constrained approach to frequency domain measurements, and preliminary results shown in Srinivasan et $\mathrm{al}^{20}$, applied in homogeneous experiments have shown substantial quantitative and qualitative improvements. Our goal in the current paper is to validate this method in tumor-simulating phantom measurements with suitable heterogeneities in different parameters. We show further the robust nature of the algorithm to increasingly noisy data, compared to the conventional technique, and apply it to measurements made on a clinical subject with fibrocystic disease (FCD). Our studies show that contrast in hemoglobin and water is observed in FCD and scatter and oxygenation may be key to studying the differences between such a case and cancer.

\section{Data Acquisition}

\section{MATERIALS AND METHODS}

The frequency domain data acquisition system is designed for cross sectional imaging of the pendant breast in three planes spaced $1 \mathrm{~cm}$ apart. A 16 source-detector fiber system for each plane is set up in a radial configuration, and is automated so that both the height and the diameter of the ring can be changed. Light signals at six wavelengths between 660 and $850 \mathrm{~nm}$ are intensity modulated at $100 \mathrm{MHz}$ and multiplexing of the source into each of the fibers is achieved by a circular translation stage. Light detection is done using high gain photo-multiplier tubes (PMTs) and heterodyning using mixers is carried out to obtain a low frequency signal $(500 \mathrm{~Hz})$ which is read by the computer. Complete details of the instrumentation can be found elsewhere ${ }^{9,21,22}$.

\section{Image Reconstruction}

Breast tissue being a highly scattering media, the diffusion approximation to the Radiative transfer equation has been used for modeling light propagation, ignoring anisotropic effects ${ }^{23}$ :

$$
-\nabla . \kappa(r) \nabla \Phi(r, \omega)+\left(\mu_{a}(r)+\frac{i \omega}{c}\right) \Phi(r, \omega)=q_{0}(r, \omega)
$$

where $\Phi(r, \omega)$ is the isotropic fluence at modulation frequency $\omega$ and position $r, \kappa(\mathrm{r})$ is the diffusion coefficient, $\mu_{a}(r)$ is the absorption coefficient, $c$ is the speed of light in the medium and $q_{0}(r, \omega)$ is an isotropic source. The diffusion coefficient can be written as

$$
\kappa(r)=\frac{1}{3\left[\mu_{a}(r)+\mu_{s}^{\prime}(r)\right]}
$$

where $\mu_{s}$ ' is the reduced scattering coefficient. The finite element method has been used to model this equation for its versatility in dealing with inhomogeneous and complicated geometries. The forward solver obtains the fluence for a given distribution of optical properties by applying suitable boundary conditions, type III (Robin-type) in our case ${ }^{24}$. In NIR tomography, the inverse problem has to be solved to obtain images of the optical properties from the measured amplitude and phase. This inverse problem is ill-posed and in order to prevent the solution from being overwhelmed by noise in the measurements, regularization techniques have been applied. An initial guess sufficiently close to the optical properties is obtained using a homogeneous pre-fitting algorithm ${ }^{22}$ and we use the Newton Raphson iterative procedure to minimize the least squares functional:

$$
\chi^{2}=\sum_{j=1}^{M}\left(\phi_{j}^{m}-\phi_{j}^{c}\right)^{2}
$$

where $\mathrm{M}$ is the total number of measurements at each wavelength, and $\phi_{j}^{m}$ and $\phi_{j}^{c}$ are the measured and calculated fluence at the boundary for each measurement point $\mathrm{j}$. The corresponding matrix equation ${ }^{24}$ is: 


$$
\left(\mathfrak{I}^{T} \mathfrak{I}+\lambda I\right) \partial \mu=\mathfrak{I}^{T} \partial \phi
$$

where $\mathfrak{I}$ is the Jacobian containing the derivatives of $\phi$ with respect to the optical properties $\mu$ given by $\left(\mu_{a}, \kappa\right)$ and $\alpha$ is the Levenberg Marquardt regularization ${ }^{25}$ parameter which is a smoothing function and keeps the solution within reasonable limits. In this manner, we obtain new search direction for update in $\left(\mu_{a}, \kappa\right)$ iteratively, the convergence criteria being $\chi^{2}$ less than $2 \%$ of that of the previous iteration, and obtain images for these optical properties.

Using the relationship from Beer's law for chromophore concentrations (c) relating to absorption, $\mu_{a}=[\varepsilon] c$, where $\varepsilon$ is the molar absorption spectra of the absorbing chromophores, the concentrations are obtained using a constrained least squares fit. The primary absorbers are oxy-hemoglobin $\left(\mathrm{HbO}_{2}\right)$, de-oxyhemoglobin $(\mathrm{Hb})$ and water, the effect of lipids being negligible since the wavelengths of use are below $850 \mathrm{~nm}$. Further, total hemoglobin is calculated as $\mathrm{Hb}_{\mathrm{T}}=\mathrm{HbO}_{2}$ $+\mathrm{Hb}\left(\right.$ in $\mu \mathrm{M}$ ), and oxygen saturation as $\mathrm{S}_{\mathrm{t}} \mathrm{O}_{2}=\mathrm{HbO}_{2} / \mathrm{Hb}_{\mathrm{T}} \times 100$ (in \%).

Similarly, for scattering the approximation to Mie theory ${ }^{26,27} \mu_{s}^{\prime}=a \lambda^{-b}$ is used to derive images of scatter amplitude (a) and scatter power $(b)$, where $\lambda$ is the wavelength in microns. Both the scattering power and amplitude depend on the scattering center size and number density and may reflect variations in breast structural composition due to different cellular, organelle and structural sizes/densities for fatty and glandular tissue.

\section{Spectral Constraints}

The spectral shapes from Beer's law and Mie theory can directly be incorporated into the reconstruction as constraints and details of this implementation are given elsewhere ${ }^{20}$. Briefly, all the wavelengths of data available are coupled together and used simultaneously in a new relationship which now minimizes the functional:

$\chi^{2}=\sum_{j=1}^{M n}\left(\phi_{j}^{m}-\phi_{j}^{c}\right)^{2}$, so that $j$ now includes all wavelength measurements $(M n)$, where $\mathrm{n}$ is the number of wavelengths available (6 in our case). The corresponding matrix equation for direct update in chromophores $\partial c$ is:

$$
\left(\mathfrak{I}^{T} \mathfrak{I}+\alpha I\right) \partial c=\mathfrak{I}^{T} \partial \phi
$$

where $\quad \partial \phi=\left(\begin{array}{l}\partial \phi_{\lambda 2} \\ . . \\ \partial \phi_{\lambda n}\end{array}\right), \mathfrak{I}=\left[\begin{array}{l}\phi_{c 1, \lambda 1} \\ \mathfrak{I}_{c 1, \lambda 2} \mathfrak{I}_{c 2, \lambda 2} \mathfrak{I}_{c 3, \lambda 2} \mathfrak{I}_{a, \lambda 2} \mathfrak{I}_{b, \lambda 2} \\ \ldots . . \\ \mathfrak{I}_{c 1, \lambda n} \mathfrak{I}_{c 2, \lambda n} \mathfrak{I}_{c 3, \lambda n} \mathfrak{I}_{a, \lambda n} \mathfrak{I}_{b, \lambda n}\end{array}\right]$ and $\partial c=\left(\begin{array}{l}\partial c_{2} \\ \partial c_{3} \\ \partial a \\ \partial b\end{array}\right)$

and the relations between $\left\lfloor\mathfrak{I}_{c, \lambda}, \mathfrak{I}_{a, \lambda}, \mathfrak{I}_{b, \lambda}\right\rfloor_{\lambda=1: n}$ and $\mathfrak{I}_{\mu_{a}}=\frac{\partial \phi}{\partial \mu_{a}}$ and $\mathfrak{I}_{\kappa}=\frac{\partial \phi}{\partial \kappa}$ have been derived in Srinivasan et $\mathrm{al}^{20}, c 1, c 2, c 3$ being the concentrations of oxy, de-oxyhemoglobin and water. Similar Levenberg-Marquardt regularization was applied as before and the computation time was approximately 25 minutes for typically 5-7 iterations, the measure of convergence being when the projection error was less than $2 \%$ of previous iteration value. The approach can easily be extended to additional wavelengths without any computational expense in the inversion process since the size of the matrix to be inverted in equation 5 depends on number of nodes (which is fixed) and not on the number of measurements, though the number of wavelengths will influence the calculation of individual Jacobian matrices. 


\section{RESULTS}

\section{Tumor-simulating Phantom results}

Amplitude and phase measurements were generated for a heterogeneous phantom using the finite element forward solver and $1 \%$ random Gaussian noise was added to mimic experimental noise 9 . This phantom was of diameter $86 \mathrm{~mm}$, and contained an inclusion of size $20 \mathrm{~mm}$, with total hemoglobin contrast of 3:1, with respect to the background. The anomaly also had lower oxygen saturation of $30 \%$ compared to the background at $60 \%$, and contrast in scatter amplitude, with value 1.5, compared to 1.2 in background. Water and scatter power were maintained homogeneous at $60 \%$ and 0.6 respectively. The spectrally constrained direct chromophore and scattering reconstruction was applied to this data. In addition, the conventional method of reconstructing at each wavelength separately and then applying least squares fit to Beer's law and Mie theory, was also applied, and Figure 1 shows the corresponding images obtained, compared to the true images in the top row. The figure shows much smoother images for all the parameters in the spectral reconstruction compared to the conventional technique. Quantitative accuracy is improved in addition to the reduced standard deviation, with spectrally constrained method giving results accurate with less than $15 \%$ error overall, and this error limit being at $43 \%$ for the conventional technique. There is also a reduction in cross-talk observed between hemoglobin and water, from $30 \%$ in conventional method to $7 \%$ in spectral; and a similar trend is seen between scatter amplitude and power as well.

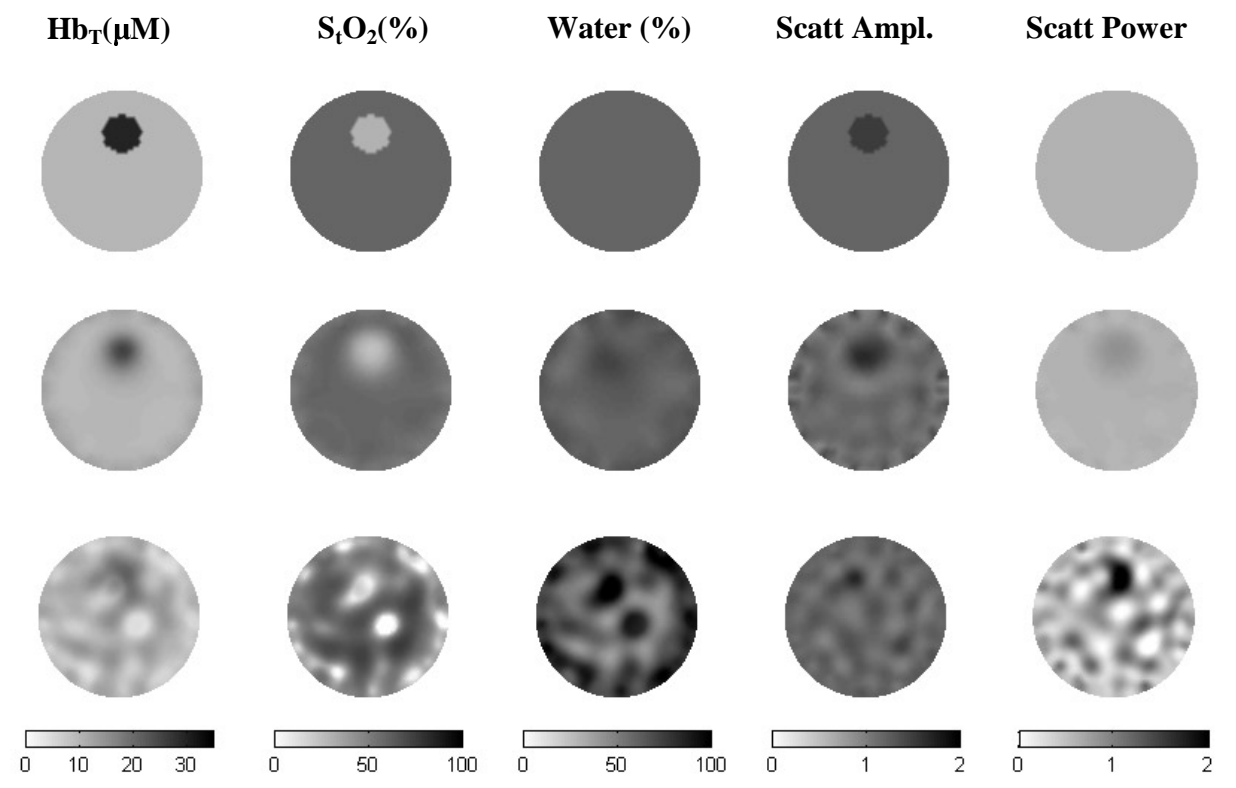

Figure 1: Recovered NIR parameter images are shown for a heterogeneous phantom with true values in the top row, as reconstructed with $1 \%$ random Gaussian noise added. The middle row shows images obtained using the spectrally-constrained reconstruction and the bottom row shows the same set of images using the conventional method of applying least squares fitting to images of absorption and reduced scattering coefficients, and then sequentially fitting for chromophore images.

Further to test the robust aspect of this algorithm, increasing amounts of random Gaussian noise from 0 to $5 \%$ in steps of $0.5 \%$ was added to this test phantom, and the mean and standard deviation in the NIR parameters as recovered from both conventional and direct spectral reconstruction have been plotted (see figure 2) alongside the true values. At $1 \%$ noise, which is the level found in our tomography system ${ }^{9}$, the reduction in standard deviations in oxygen saturation, water and scatter power are most striking. The trend is continued at 5\% noise in the amplitude and phase data (5\% is possibly the limit of noisy data found typically in such measurement systems). The spectrally constrained technique still gives quantification accurate within $15 \%$ of true values overall, but this is not the case in the conventional method, where the high standard deviations in the images make it impossible to obtain useful NIR information. 

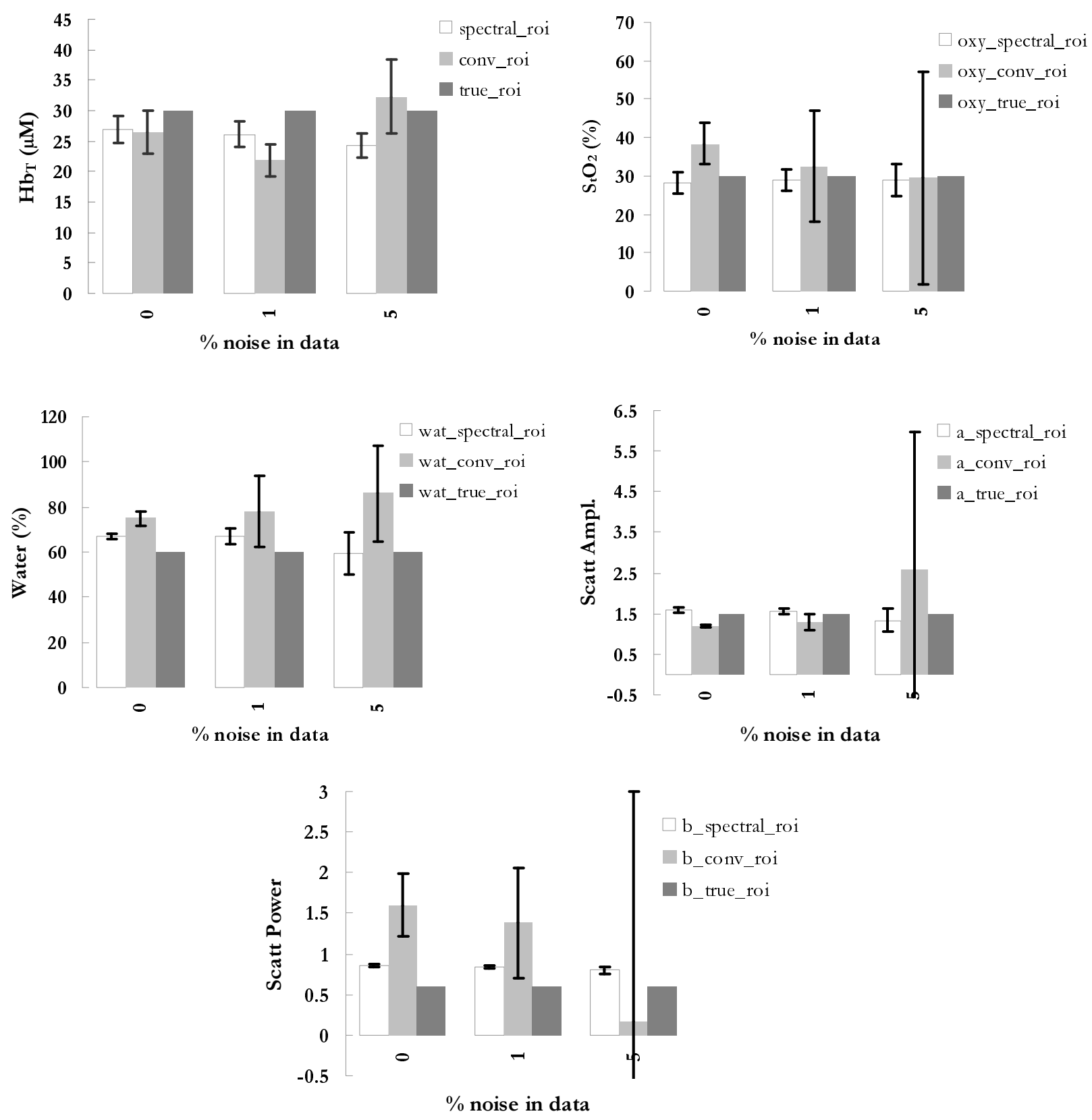

Figure 2: Mean and standard deviations from the images recovered are shown, using both spectrally constrained reconstruction and the conventional method, for the test phantom used in Figure 1, with increasing amounts of random Gaussian noise as the abscissa. The results are average values taken from the region of the heterogeneity, for (a) $\mathrm{Hb}_{\mathrm{T}}$ in $\mu \mathrm{M}$, (b) $\mathrm{S}_{\mathrm{t}} \mathrm{O}_{2}$ in $\%$, (c) water in $\%$, (d) scatter amplitude and (e) scatter power. The background values were observed to stay constant for both methods.

\section{Clinical Case: Fibrocystic Disease (FCD)}

Frequency domain measurements were obtained from a subject with an $8 \mathrm{~mm}$ tumor, diagnosed as FCD, in the left breast. The total breast diameter was measured to be $102 \mathrm{~mm}$ in the plane 1, where the location of cyst was marked, and given by the Radiologist as upper inner quadrant, 11.00-12.00 clock position. Results from the previous section and as 
documented ${ }^{20}$ suggest that the spectrally constrained technique is significantly superior to the conventional method in both quantitative and qualitative aspects, and hence only the NIR parameter images obtained using this reconstruction have been shown here (see figure 3). The tumor is visible in the 12.00 position in the total hemoglobin and water images. There is a contrast of 1.25:1 in the $\mathrm{Hb}_{\mathrm{T}}$ image, between the maximum in the tumor and background mean, which is much lower than that typically found in malignancies. In addition, no decrease in oxygenation is observed, with this parameter remaining almost homogeneous. Water shows an increase of up to a maximum value of $85 \%$ at the site of the tumor, which corresponds well with theory that FCDs may contain fluid filled cysts. Since the physiology of this disease is complex, and may vary from distinct masses to diffuse masses and/or palpable lesions ${ }^{28}$, the additional increases in hemoglobin and water found close to the tumor may very well be physiologically true. The radiodensity category of the breast of this subject was given to be fatty/scattered type with less of glandular tissue, and the recovered background hemoglobin $(\sim 10 \mu \mathrm{M})$ agrees well with the expected lower vascularity in such tissue. In addition, the low values of scatter power correspond well with a previous hypothesis that scattering may be a non-invasive indicator of radio-density ${ }^{4}$.

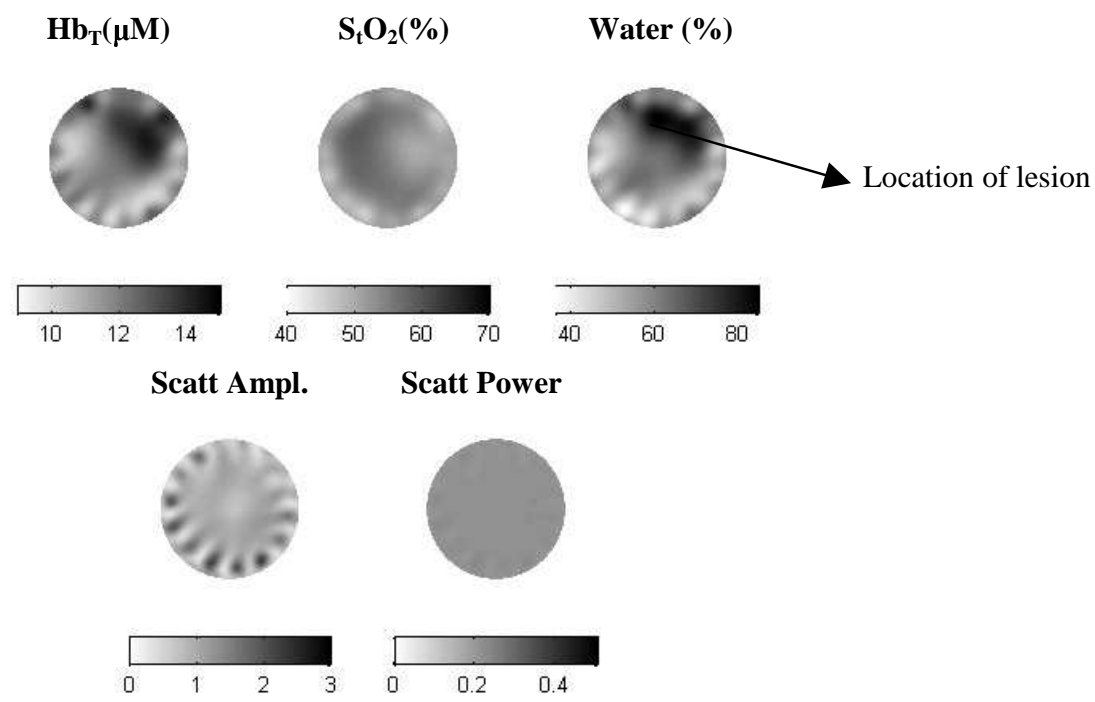

Figure 3: Recovered images for the NIR parameters are shown as reconstructed with the spectrally-constrained reconstruction, using data collected from a subject with a localized area of fibrocystic disease of size 8mm, at the 12:00 o'clock position of this circular cranocaudal view. Contrast is observed at location of the lesion, in total hemoglobin and water, and no decrease in oxygen saturation is observed.

\section{DISCUSSION}

The approach of using spectrally-constrained direct chromophore and scatter tomography using frequency domain measurements has been validated here, for a heterogeneous phantom simulation with $1 \%$ random Gaussian noise. The inclusion of size $20 \mathrm{~mm}$ contained contrast in total hemoglobin and scatter and lower oxygen saturation corresponding to malignancies ${ }^{28}$. Reconstruction was carried out by both the spectral technique, and the conventional method involving single wavelength optical properties recovery, and the images recovered were compared to the true images in figure 1. Results showed substantial improvement in the image quality with suppression of noise and image artifacts, using the new technique. Quantification was also improved with the recovered mean values for the NIR parameters in the anomaly accurate within $85 \%$ of true values.

The significant reduction in the crosstalk between oxyhemoglobin and water can be explained by the nature of the chromophore and scattering spectra. Water and oxy-hemoglobin show similar behavior in the $600-800 \mathrm{~nm}_{\text {range }}^{3}$, and the water characteristics which help separate the two appear only at longer wavelengths, in our case $849 \mathrm{~nm}$. In the spectrally-constrained reconstruction, this being apriori information, the cross-talk between the two, and hence the noise in water images is significantly suppressed. In the conventional technique, this cross-talk over-rides the reconstruction, affecting the accuracy and quality of water images. Similarly, both the de-oxyhemoglobin and Mie scattering follow a 
decreasing trend, making it difficult to separate the two in the conventional technique. However, since the specific trends of these are applied as constraints in the new method, significantly more robust scatter images result. These constraints are also responsible for the robust nature of the algorithm to higher levels of noise as compared to before, and this has been tested out for up to 5\% random Gaussian noise in the measurements of amplitude and phase. Figure 2 shows that the mean values for this level of noise are quantitatively accurate and show significant reduction in the standard deviations in the region of the heterogeneity.

Finally, the method has been used to characterize fibrocystic disease using clinical data from a subject with an $8 \mathrm{~mm}$ tumor. FCDs cover a broad range of alterations from diffuse masses to distinct tumors and frequently, many of these tumors with ductal proliferation mimic cancers making diagnosis difficult ${ }^{28}$. By understanding the NIR parameter images for this disease, it may be possible to overcome this limitation. In the images we have recovered here using the spectrally constrained technique, contrast in total hemoglobin and water at the location of the tumor was observed. The increase in hemoglobin $(13.8 \mu \mathrm{M}$ maximum in region of tumor, compared to $11 \mu \mathrm{M}$ in background) is lower than typical of cancers, and the contrast in water agrees well with theoretical predictions of the physiology of this benign disease ${ }^{28}$. No decrease in oxygenation is observed and this is the distinguishing parameter between FCDs and cancers. We are currently following this trend in further clinical cases, and the results shown here and in progress hold promise in understanding the nature of NIR information.

\section{ACKNOWLEDGEMENTS}

The authors would like to acknowledge collaboration from Steven P. Poplack, Sandra Soho and Christine Kogel in carrying out the clinical examinations. This work has been supported through NIH grants PO1CA80139 and RO1CA69544 as well as DAMD17-03-01-0405.

\section{REFERENCES}

1. F.F. Jobsis, "Non-invasive, infra-red monitoring of cerebral and myochardial oxygen sufficiency and circulatory parameters." Science, vol. 198 pp. 1264-1267.1977.

2. $\quad$ B.W. Pogue, S.P. Poplack, T.O. McBride, W.A. Wells, O.K. S., U.L. Osterberg, and K.D. Paulsen, "Quantitative Hemoglobin Tomography with Diffuse Near-Infrared Spectroscopy: Pilot Results in the Breast." Radiology, vol. 218(1) pp. 261-6.2001.

3. B.J. Tromberg, N. Shah, R. Lanning, A. Cerussi, J. Espinoza, T. Pham, L. Svaasand, and J. Butler, "Noninvasive in vivo characterization of breast tumors using photon migration spectroscopy." Neoplasia (New York), vol. 2(1-2) pp. 26-40.2000.

4. S. Srinivasan, B.W. Pogue, S. Jiang, H. Dehghani, C. Kogel, S. Soho, J.J. Gibson, T.D. Tosteson, S.P. Poplack, and K.D. Paulsen, "Interpreting hemoglobin and water concentration, oxygen saturation and scattering measured in vivo by near-infrared breast tomography." PNAS, vol. 100(21) pp. 12349-12354.2003.

5. $\quad$ M. Hockel, P.Vaupel, "Tumor Hypoxia : Definitions and Current Clinical, Biologic, and Molecular Aspects." J. Natl. Cancer Inst., vol. 93(4) pp. 266-276.2001.

6. D. Grosenick, H. Wabnitz, K.T. Moesta, J. Mucke, M. Moller, C. Stroszczynski, J. Stossel, B. Wassermann, P.M. Schlag, and H. Rinneberg, "Concentration and oxygen saturation of haemoglobin of 50 breast tumours determined by time-domain optical mammography." Phys Med Biol, vol. 49(7) pp. 1165-81.2004.

7. J.G. Elmore, M. B. Barton, V. M. Moceri, S. Polk, P. J. Arena and S. W. Fletcher, "Ten-Year Risk of False Positive Screening Mammograms and Clinical Breast Examinations." The New England Journal of Medicine, vol. 338(16) pp. 1089-1096.1998.

8. M.S. Patterson, C. B., and B.C. Wilson, "Time resolved reflectance and transmittance for the non-invasive measurement of tissue optical properties." Appl. Opt., vol. 28 pp. 2331-2336.1989.

9. T.O. Mcbride, B.W. Pogue, S. Jiang, U.L. Osterberg, and K.D. Paulsen, "A parallel-detection frequencydomain near-infrared tomography system for hemoglobin imaging of the breast in vivo." Review of Scientific Instruments, vol. 72(3) pp. 1817-1824.2001.

10. M.G. Nichols, Hull, E. L. and Foster, T. H., "Design and testing of a white-light, steady-state diffuse reflectance spectrometer for determination of optical properties of highly scattering systems." Applied Optics, vol. 36(1) pp. 93-104.1997.

11. S.R. Arridge and M. Schweiger, "Image reconstruction in optical tomography." Phil. Trans. R. Soc. Lond. B, vol. 352 pp. 717-726.1997. 
12. B.J. Tromberg, Coquoz, O., Fishkin, J. B., Pham, T., Anderson, E. R., Butler, J., Cahn, M., Gross, J. D., Venugopalan, V., Pham, D., "Non-invasive measurements of breast tissue optical properties using frequencydomain photon migration." Phil. Trans. R. Soc. Lond. B, vol. 352 pp. 661-668.1997.

13. T.O. McBride, B.W. Pogue, S. Jiang, U.L. Osterberg, K.D. Paulsen, and S.P. Poplack, "Multi-spectral nearinfrared tomography: a case study in compensating for water and lipid content in hemoglobin imaging of the breast." J. Biomed. Opt., vol. 7 pp. 72-79.2002.

14. T. Durduran, R.Choe, J.P.Culver, L.Zubkov, M.J.Holboke, J.Giammarco, B.Chance and A.G.Yodh, "Bulk optical properties of healthy female breast tissue." Phys. Med. Biol., vol. 47 pp. 2847-2861.2002.

15. S. Srinivasan, Pogue, B. W., Dehghani, H., Jiang, S., Song, X. and Paulsen, K. D., "Improved quantification of small objects in near-infrared diffuse optical tomography." Journal of Biomed Opt., vol. 9(6) pp. 116171.2004.

16. X. Song, B.W. Pogue, S. Jiang, M.M. Doyley, H. Dehghani, T.D. Tosteson, and K.D. Paulsen, "Automated region detection based on the contrast-to-noise ratio in near-infrared tomography." Appl Opt, vol. 43(5) pp. 1053-62.2004.

17. E. Heffer, V. Pera, O. Schutz, H. Siebold, and S. Fantini, "Near-infrared imaging of the human breast: complementing hemoglobin concentration maps with oxygenation images." Journal of Biomed Opt., vol. 9(6) pp. 1152-60.2004.

18. A. Corlu, T. Durduran, R. Choe, M. Schweiger, E.M. Hillman, S.R. Arridge, and A.G. Yodh, "Uniqueness and wavelength optimization in continuous-wave multispectral diffuse optical tomography." Opt Lett, vol. 28(23) pp. 2339-41.2003.

19. A. Li, Q. Zhang, J.P. Culver, E.L. Miller, and D.A. Boas, "Reconstructing chromosphere concentration images directly by continuous-wave diffuse optical tomography." Opt Lett, vol. 29(3) pp. 256-8.2004.

20. S. Srinivasan, B.W. Pogue, S. Jiang, H. Dehghani, and K.D. Paulsen, "Spectrally Constrained Chromophore and Scattering NIR Tomography Provides Quantitative and Robust Reconstruction." Applied Optics.2005-in press.

21. B.W. Pogue, M. Testorf, T. McBride, U. Osterberg, and K. Paulsen, "Instrumentation and design of a frequency-domain diffuse optical tomography imager for breast cancer detection." Opt. Express, vol. 1(13) pp. 391-403.1997.

22. T.O. McBride, B.W. Pogue, U.L. Österberg, and K.D. Paulsen, "Strategies for Absolute Calibration of Near Infrared Tomographic Tissue Imaging", in Oxygen Transport to Tissue XXI, J.F. Dunn, Swartz, H. M., Editor. 2001, Lengerich: Pabst.

23. A. Ishimaru, Wave propagation and scattering in random media. Vol. 1. 1978: Academic Press, Inc., New York.

24. K.D. Paulsen, and Jiang, H., "Spatially varying optical property reconstruction using a finite element diffusion equation approximation." Med. Phys., vol. 22(6) pp. 691-701.1995.

25. D.W. Marquardt, "An algorithm for least squares estimation of nonlinear parameters." J. Soc. Ind. Appl. Math., vol. 11 pp. 431-441.1963.

26. H.J. van Staveren, C.J.M. Moes, J. van Marle, S.A. Prahl, and M.J.C. van Gemert, "Light scattering in intralipid-10\% in the wavelength range of 400-1100 nm." Applied Optics, vol. 30(31) pp. 4507.1991.

27. J.R. Mourant, T. Fuselier, J. Boyer, T.M. Johnson, and I.J. Bigio, "Predictions and measurements of scattering and absorption over broad wavelength ranges in tissue phantoms." Applied Optics, vol. 36(4) pp. 949.1997.

28. S. Thomsen, and D. Tatman, "Physiological and Pathological Factors of Human Breast Disease That can Influence Optical Diagnosis." Ann. N. Y. Acad. Sci., vol. 838 pp. 171-193.1998. 